\title{
$\mathrm{AHP}$ 기반 $\mathrm{PL}$ 상품 업체 평가모델
}

최소영 ${ }^{1}$, 김용민 ${ }^{1}$, 이홍철 ${ }^{*}$

${ }^{1}$ 고려대학교 산업경영공학과

\section{An assessment model for PL-product companies based on the Analytic Hierarchy Process}

\author{
So-Young Choi ${ }^{1}$, Yong-Min $\mathrm{Kim}^{1}$ and Hong-Chul Lee ${ }^{1}$ \\ ${ }^{1}$ Department of Industrial Management Engineering, Korea University
}

\begin{abstract}
요 약 본 연구는 PL(Private Level)상품 업체 선정을 위한 평가모델을 다룬다. 제안하는 평가모델은 AHP(Analytic Hierarchy Process)를 기반으로 다수의 평가항목에 대한 가중치를 도출한다. 평가항목은 국내 대형마트에서 활용하는 실 업체 선정기준으로, 특히 매출 비중이 높은 식품품목을 대상으로 한다. 식품품목에 대한 54 개의 평가항목을 4단계 로 계층화 하며, 이를 기반으로 해당 업계 전문가들을 대상으로 한 설문을 수행한다. 설문을 통해 형성한 쌍대비교를 활용하여 평가항목 별 가중치를 추정하며, 이를 실 산업에서 활용 가능한 정량적 대안으로서 제안한다. 마지막으로, 제안하는 평가모델과 기존의 단순합산방식을 비교하는 평가모델 검증을 포함한다. 연구를 통해 PL상품 업체 선정에 중요도가 높은 요소로 $\mathrm{HACCP}$ 검증 및 유효성 평가 (0.279281), 운송(원료 입고 및 제품 출하) (0.117706), 법규 준수 중점 관리 (0.066392) 를 선별하였다. 제안하는 평가모델은 기존방식으로 우위를 판단하기 힘든 대안들에 대하여 평가 항목의 중요도를 반영함에 따라 업체 선정이 용이한 결과를 제시함을 검증하였다.
\end{abstract}

\begin{abstract}
This paper addresses an assessment model for selecting PL(Private Level) product companies. The proposed model extracts the weights of evaluation elements based on AHP(Analytic Hierarchy Process). These evaluation elements are from real-world instances in a domestic hyper market. Especially, the model points at food products which constitute a large portion of entire profits in the market. In this model, we first classify the 54 evaluation elements into 4 layers and secondly carry out a survey with relevant specialists based on them. We also estimate the weights of evaluation elements according to pairwise comparisons from the survey, and propose them as a quantitative alternative which can be applied in real-world problems. Finally, the pilot-study is conducted to compare the proposed model with the existing simple summation method. From this study, HACCP system assessment and Review(0.279281), Transportation(0.117706) and Fundamental law observance $(0.066392)$ are presented as the key evaluation elements for selecting PL product companies. The proposed model facilitates the company selection among those candidate companies which is not easy to determine the superiority by reflecting the importance of evaluation elements.
\end{abstract}

Key Words : Analytic Hierarchy Process, Assessment Model, Private Level Product

1. 서론

유통업체에서는 브랜드에 대한 책임의 주체에 따라 제 조업체 브랜드( $\mathrm{NB}$, National Brand)와 유통업체 브랜드
(PL, Private Label)로 구분한다[1]. PB(Private Brand)라고 도 불리는 $\mathrm{PL}$ 은 제조업체가 소유권을 갖는 $\mathrm{NB}$ 와는 달리 유통업체가 브랜드의 소유권을 갖는 것이 가장 큰 특징 이다. 이는, 유통업체가 독자적 혹은 제조업체와 제휴하

본 논문은 2013년도 3단계 BK21PLUS 사업에 의하여 지원되었음.

${ }^{*}$ Corresponding Author : Hong-Chul Lee(Korea Univ.)

Tel: +82-3-290-3389 email: hclee@korea.ac.kr

Received September 24, 2013 Revised (1st November 20, 2013, 2nd December 17, 2013)

Accepted January 9, 2014 
여 개발한 상품을 유통업체의 단독 브랜드로 판매 또는 서비스함을 의미한다[2,3].

PL상품은 높은 수익률, 제조업체와의 협상수단 및 유 통업체에 대한 충성도 형성과 같은 이점 때문에 북미 및 서유럽 시장에서 빠른 속도로 성장하고 있다[4]. 또한, 최 근에는 대형 유통업체의 가격파괴 및 공격적 경영에 의 해 관심이 증대되고[5], 소비자의 소득변화와 소비패턴 변화에 탄력적으로 대응하기 위해 PL상품의 제품개발과 판매시장 확대됨에 따라 그 중요성이 강조되고 있다[6].

Table 1은 주요 대형마트의 PL상품 매출 상위 품목 현 황으로 2011년 상반기 기준 신선식품 $40.3 \%$, 가공식품 $27.0 \%$, 위생용품 $7.7 \%$ 순으로 나타난다. 특히 신선식품 과 가공식품을 포함하는 식품품목의 비중이 $67.3 \%$ 로, 식 품품목이 주요 PL상품임을 알 수 있다.

[Table 1] Current state of PL products high on the list based on sales

[derived from each enterprise in June, 2011]

\begin{tabular}{c|c|c}
\hline Item & $\begin{array}{c}\text { The number of } \\
\text { products }\end{array}$ & $\begin{array}{c}\text { Ratio } \\
(\%)\end{array}$ \\
\hline \hline Fresh food & 121 & 40.3 \\
\hline Processed food & 81 & 27.0 \\
\hline Hygienic goods & 23 & 7.7 \\
\hline Home appliances & 20 & 6.7 \\
\hline Bedding & 13 & 4.3 \\
\hline Detergent & 7 & 2.3 \\
\hline Kitchen utensils & 6 & 2.0 \\
\hline Disposable product & 5 & 1.7 \\
\hline Bath supplies & 5 & 1.7 \\
\hline Office supplies & 4 & 1.3 \\
\hline
\end{tabular}

PL상품 관련연구는 크게 네 가지 분야로 분류된다. 첫 번째는 마케팅 전략 분야, 두 번째는 소비자 관련 분야, 세 번째는 광고 분야, 네 번째는 제조업체 관련 분야이다. 기존의 PL상품에 대한 연구는 마케팅 전략 분야에서 활 발히 진행되었다. 그러나 유통업체가 PL상품에 대한 수 익을 극대화하기 위해서는 제조업체에 대한 관리도 중요 함에도 불구하고 이에 대한 연구는 상대적으로 부족한 것이 현실 이다[7,8].

PL상품 제조업체 관련 연구로 유통업체와 제조업체 간 제휴에 관한 연구[9-11] 및 점포 충성도에 관한 연구 [4] 등이 있다. 제조업체 관련 분야에서 업체선정은 PL상 품을 효과적으로 관리하기 위한 중요한 요건 중 하나로 인식된다. 제조업체 선정에 관한 연구로 Kumar 등 [12] 은 PL상품 업체 선정에 대한 연구를 다루었다. Kumar 등
은 고객의 가치 및 마진률에 따라 NB상품을 보유한 제조 업체에게 PL상품을 위탁하는 것이 최적임을 보였지만, 업체선정을 위한 정량적 기준을 설정하는 데에 한계가 있다.

국내 대형마트의 PL상품 업체 선정 방식은 동종 제조 업계 2,3,4위 업체를 대상으로 심사하여 최고점 평가를 받은 업체를 PL상품 제조업체로 선정한다. 상대적으로 판매율이 높은 1 위 제조업체는 대부분 PL 상품의 필요성 을 인식하지 못해 협상하지 않는다. 국내 대형마트 PL상 품 중 매출이 높은 식품품목 업체선정에 대한 실 평가항 목은 Table 2 와 같다. 각 평가 항목에 대해 5 단계 등급으 로 평가하여 합산하고, 최종 평가점수를 총 1000 점으로 환산한다. 이러한 방식으로 평가할 경우, 각 항목에 대한 단순합산방식으로 평가가 이루어지고 있음에 따라 항목 간의 상대적 중요도를 반영하는데 어려움이 따른다.

제안하는 방법론은 AHP(Analytic Hierarchy Process) 를 기반으로 하며, PL상품 중 가장 큰 매출 비중을 차지 하는 식품 관련 업체에 초점을 맞춘다. 연구에 대한 사례 분석은 실 업체에 대하여 진행하며, 해당 업체에서 활용 하는 평가항목과 전문가 설문을 바탕으로 각 평가항목의 상대적 중요도를 제시한다. 연구를 통해 기존의 단순 합 계 방식을 벗어나 평가항목의 중요도를 반영하는 정량적 방법론을 제시할 것으로 기대한다.

현재 국내 대형마트의 PL상품 업체 선정에 관한 연구 는 전무한 실정이다. 따라서 본 연구에서는 국내 PL상품 업체 선정에 대해 AHP를 기반으로 한 정량적 방법론을 제시하여, 그동안 활발하지 못했으나 이제 막 PL상품 업 체 선정에 관심을 가지고 시작단계에 있는 우리나라의 $\mathrm{PL}$ 상품 업체 선정 관련 연구에 초석이 되고자 한다.

\section{2. 업체 선정 관련 연구}

업체 선정을 위한 방법론으로는 선형계획법(Linear Program), 다목적계획법(Multi-objective program), 시뮬레 이션(Simulation), AHP(Analytic Hierarchy Process), DEA (Data Envelopment Analysis) 등을 활용할 수 있다.

그 중에서도 AHP는 계층화 및 쌍대비교 설문을통해 전문가 설문의 직관성을 증대하고, 다수의 의사결정 기준 에 대한 가중치를 형성할 뿐만 아니라 또한 $\mathrm{CR}$ 계수 평가 를 통해 설문의 타당성을 검증할 수 있는 데에 강점이 있 다. $\mathrm{AHP}$ 는 전문가 설문을 기반으로 다기준 의사결정문 제의 평가항목에 대한 가중치를 산출하는 방법으로 일반 적으로 계층화, 쌍대비교, 가중치 추정, 가중치 통합 및 일관성 검증으로 구성된다[13]. 
[Table 2] existing method for selecting a PL product company

\begin{tabular}{|c|c|c|}
\hline \multirow{2}{*}{ Factors } & \multicolumn{2}{|c|}{ Simple Summation } \\
\hline & Score & Final Score \\
\hline Fundamental Law Observance & $\begin{array}{l}\text { a1 } \\
\end{array}$ & \multirow{54}{*}{ 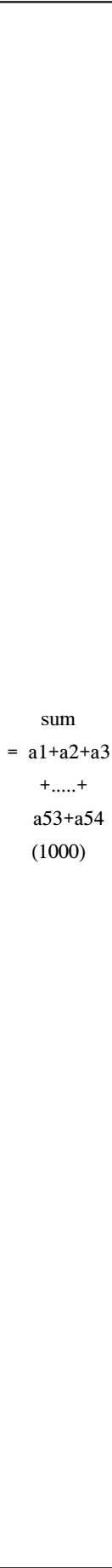 } \\
\hline Law Observance & $\mathrm{a} 2$ & \\
\hline Morality & a3 & \\
\hline Surroundings & $\mathrm{a} 4$ & \\
\hline Working Area & a5 & \\
\hline Floor, Wall, Ceiling & a6 & \\
\hline Subsidiary Facility & a7 & \\
\hline Drainage and Piping & a8 & \\
\hline Exit and Passage & a9 & \\
\hline Window & a10 & \\
\hline Illumanation & a11 & \\
\hline Temperature, Humidity & a12 & \\
\hline Ventilation & a13 & \\
\hline Insect Proof & a14 & \\
\hline Worker Health Care & a15 & \\
\hline Individual Health Control & a16 & \\
\hline Process Contamination & a17 & \\
\hline Waste Management & a18 & \\
\hline Disinfection & a19 & \\
\hline Machinery Management & a20 & \\
\hline Refrigeration Plant & a21 & \\
\hline Transportation & a22 & \\
\hline Supply Management & $\mathrm{a} 23$ & \\
\hline Storage & a24 & \\
\hline Facility Inspection & a25 & \\
\hline Product Designing & a26 & \\
\hline Packing & a27 & \\
\hline Water Management & a28 & \\
\hline Product Inspection & a29 & \\
\hline Recall Program & a30 & \\
\hline Foreign Material & a31 & \\
\hline Defective Product & a32 & \\
\hline System Construction & a33 & \\
\hline Preceding Requisite & a34 & \\
\hline Product Characteristic & a35 & \\
\hline Process Flow Diagram & a36 & \\
\hline Hazard Analysis & a37 & \\
\hline Critical Control Point & a38 & \\
\hline Threshold Level & a39 & \\
\hline Monitoring & $\mathrm{a} 40$ & \\
\hline HACCP Correction Measure & a41 & \\
\hline Verification & a42 & \\
\hline Documentation & a43 & \\
\hline System Examination & a44 & \\
\hline Quality Policy and Goals & $\mathrm{a} 45$ & \\
\hline Quality Manual & a46 & \\
\hline Responsibility and Authority & a47 & \\
\hline Standard of Goods & a48 & \\
\hline Document Control & a49 & \\
\hline Maintenance of Record & a50 & \\
\hline Process Control & a51 & \\
\hline Correction Measure & a52 & \\
\hline Complaint Handling & a53 & \\
\hline Instruction and Training & a54 & \\
\hline
\end{tabular}

$\mathrm{AHP}$ 기반 업체 선정에 관한 연구는 다음과 같다. 김대 기 [14]는 AHP를 활용하여 물류업체의 선정 우선순위를 설정하였고, 최영윤 [15]은 전자상거래 업체에서 발생하 는 택배업체 선정에 대한 연구를 다루었다. 최세종 [16] 은 항공기 엔진수리 업체 선정에 관한 연구를 제시하였 으며, 김승렬 [17]은 소프트웨어 외주업체 선정방안에 대 해 연구하였다.

\section{3. 모델 연구}

제 2장에서 설명된 AHP 방법론을 기반으로 PL상품 업체 선정 모델을 제시한다. 특히 PL상품 중 가장 큰 매 출 비중을 차지하는 식품 관련 업체에 초점을 맞추며, 실 업체에서 사용하는 평가항목을 활용한다. 평가항목을 계 층화하고, 전문가 설문을 통해 쌍대비교행렬을 구성하여, 평가항목의 가중치와 종합 가중치를 산출한다.

\section{1 평가항목 구성 및 계층화}

$\mathrm{AHP}$ 는 의사결정문제를 상호 관련된 의사결정 사항들 의 계층으로 분류하여 의사결정계층-의사결정모형을 설 정한다[18]. 본 연구에서 사용하는 AHP 평가항목은 국내 최대 대형마트의 식품업체 선정 시 고려되는 실 평가 기 준을 이용하며, 이를 계층화 하는 과정은 10 년 이상 경력 의 PL상품 업체 선정 전문가에 의해 이루어진다. Fig. 1 에서와 같이 총 54 개 평가항목을 4단계로 계층화하며, 1 단계에서 경영적 요인, 환경적 요인 및 품질적 요인으로 구분한다. 경영적 요인은 각 항목을 2 단계로 구성하고 환 경적 요인과 품질적 요인은 각 항목을 4단계로 구성한다.

\subsection{1 경영적 요인}

경영적 요인은 법규준수 중점관리, 법규준수 일반 및 윤리경영을 평가항목으로 포함한다. 법규준수 중점관리 는 자가품질검사, 원산지, 유통기한 및 원료에 대한 적법 성을 평가한다. 법규준수 일반은 무게·용량·개수의 허용 오차, 행정처분 및 위해식품 관리에 대한 적법성을 평가 한다. 윤리경영은 위험물 안전 관리, 응급 대응책 관리 및 작업시간·임금 관리에 대한 적법성을 평가한다.

\subsection{2 환경적 요인}

환경적 요인은 작업장 환경, 위생 관리, 제조시설 및 자재관리로 구성한다. 작업장환경은 물리적 작업장 환경 과 순환적 작업장 환경으로 구성하며, 물리적 작업장 환 경은 작업장주변, 작업장, 건물바닥·벽·천장 및 부대시설 


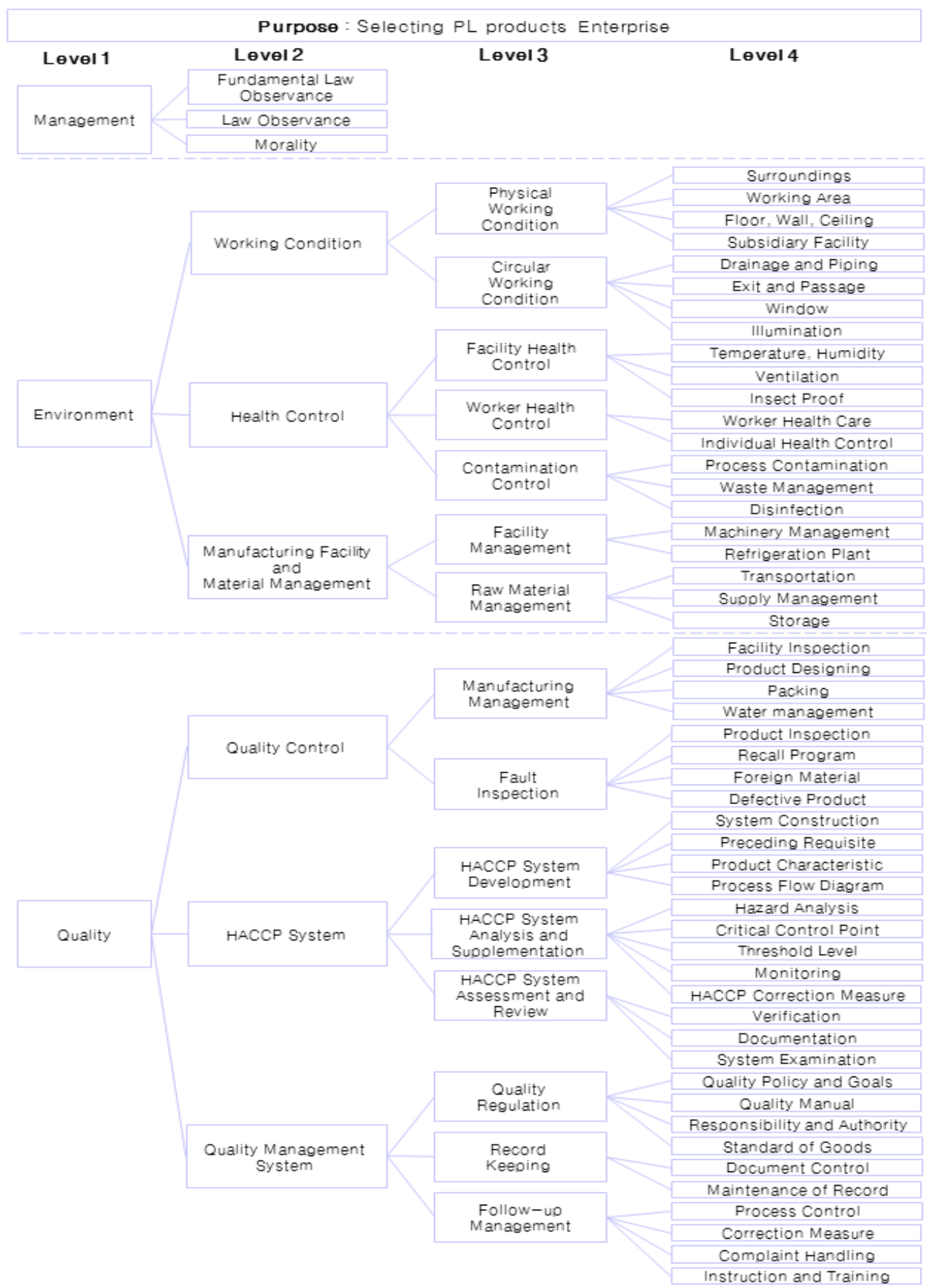

[Fig. 1] The structure of factors for evaluating PL products enterprise

을 평가항목으로 포함하고, 순환적 작업장 환경은 배수 및 배관, 출입구 및 통로, 창, 채광 및 조명을 평가항목으 로 포함한다.

위생관리는 시설 위생관리, 작업자 위생관리 및 오염
물 위생관리로 구성한다. 시설 위생 관리는 온도·습도 관 리, 환기시설 관리, 방충·방서 관리를 평가항목으로 포함 하고, 작업자 위생관리는 종업원 건강관리, 개인위생 관 리를 평가항목으로 포함하며, 오염물 위생관리는 동선계 
획 및 공정간 오염방지, 폐기물관리, 세척·소독 관리를 평 가항목으로 포함한다.

제조시설 및 자재관리는 설비관리, 원료 및 자재관리 로 구성한다. 설비관리는 제조시설 및 기계 기구류 등 설 비관리, 냉장.냉동 설비 관리를 평가항목으로 포함하며, 원료 및 자재관리는 운송(원료 입고 및 제품 출하), 구매 및 공급자 관리, 보관 식별 및 추적성을 평가항목으로 포 함한다.

\subsection{3 품질적 요인}

품질적 요인은 제품관리, $\mathrm{HACCP}$ 시스템, 품질경영 시 스템으로 구성한다. 제품관리는 생산 및 제조 관리와 불 량 검사 및 관리로 구성한다. 생산 및 제조 관리는 시설. 설비·기구 등검사, 제품설계 및 개발, 포장, 용수관리를 평가항목으로 포함하고, 불량 검사 및 관리는 제품검사, 회수프로그램관리, 이물질 관리, 부적합품 관리를 평가항 목으로 포함한다.

$\mathrm{HACCP}$ 시스템은 HACCP 시스템 개발, HACCP 시 스템 분석 및 보완, $\mathrm{HACCP}$ 시스템 평가 및 검토로 구성 한다. HACCP 시스템 개발은 $\mathrm{HACCP}$ 시스템 구축, 선행 요건 프로그램, 제품 특성, 제조공정 흐름도를 평가항목 으로 포함하고, $\mathrm{HACCP}$ 시스템 분석 및 보완은 위해요소 분석, 중요 관리점, 한계기준, 모니터링, $\mathrm{HACCP}$ 시정조 치를 평가항목으로 포함하며, $\mathrm{HACCP}$ 시스템 평가 및 검 토는 검증 및 유효성 평가, 문서화 및 기록 관리, $\mathrm{HACCP}$ 시스템 검토를 평가항목으로 포함한다.

품질경영 시스템은 품질 규약, 기록 관리, 사후관리로 구성한다. 품질 규약은 품질방침 및 목표, 품질 매뉴얼, 책임과 권한, 제품 규격을 평가항목으로 포함하며, 기록 관리는 문서관리, 기록 유지를 평가항목으로 포함하고, 사후 관리는 공정 관리, 시정 조치, 불만 처리, 교육 및 훈 련을 평가 항목으로 포함한다.

\section{2 항목별 가중치 형성}

쌍대비교행렬 $A$ 의 고유벡터는 Saaty가 제안한 누승법 (Power method)을 이용하여, 최대 고유값에 해당하는 고 유벡터를 각 평가항목의 가중치 벡터 $W$ 로 계산한다. 해 당 업체 전문가 3 명의 설문조사를 통해 쌍대비교행렬을 구성하고, 쌍대비교행렬은 AIJ(Aggregation of Individ -ual Judgments)기법으로 통합한다. AIJ 기법은 다수의 설 문조사 결과를 통합하는 방법론 중 하나로 쌍대비교행렬 의 기하평균값을 활용한다.

Table 3 은 전문가 설문조사 통해 산출한 그룹별 상대 적 가중치를 나타낸다. 상대적 중요도 측정 결과, 최상위 단계에서는 품질적 요인(0.566)이 경영적 요인, 환경적
요인 보다 중요도가 높게 나타났다. 품질적 요인 중에서 는 $\mathrm{HACCP}$ 시스템(0.806)이 제품관리, 품질경영 시스템 보다 중요하게 나타났으며, $\mathrm{HACCP}$ 시스템에서는 $\mathrm{HACCP}$ 시스템 평가 및 검토(0.793)가 HACCP 시스템 개발, $\mathrm{HACCP}$ 시스템 분석 및 보완보다 중요도가 높게 나타났다. 마지막으로, $\mathrm{HACCP}$ 시스템 평가 및 검토에서 는 $\mathrm{HACCP}$ 에 대한 검증 및 유효성 평가(0.772)가 $\mathrm{HACCP}$ 에 대한 문서화 및 기록관리, $\mathrm{HACCP}$ 시스템 검 토 보다 중요하게 나타났다.

\section{3 평가모델 제시}

PL상품 업체 선정을 위한 최종 점수는 Bottom-up 방 식으로 산출한다. Fig 2.와 같이 가장 하위단계인 Level 4 요인에 대한 평가 점수로부터 Level 3, Level 2, Level 1 을 거쳐 최종 점수를 순차적으로 계산한다. 최하위 단계 에 해당하는 Level 4 평가항목은 실제 PL상품 업체 선정 을 담당하는 전문가들이 점수를 책정한다. 상위 요인들은 각 요인이 포함하고 있는 하위 평가항목 점수의 가중산 술평균으로 도출한다.

Table 3에 제시된 가중치를 기반으로 한 최종 PL업체 선정 평가 모델은 Table 4 와 같다. 최종 평가점수 산출을 위해 평가항목 별 전문가 평가점수를 Table 4 의 a1 a54 에 입력한다. 각 PL상품 업체들에 대한 각 평가항목의 입 력점수는 일반적으로 전문가에 의해 평가되나 이 역시 $\mathrm{AHP}$ 방법론을 활용하여 산출 가능하다.

각 단계에 대한 평가점수는 Table 4에 제시한 수식에 의해 순차적으로 산출한다. Level 4 에 대한 평가 점수 a1 $\sim \mathrm{a} 54$ 를 기반으로 Level 3 의 평가점수 $\mathrm{b} 1 \sim \mathrm{b} 15$ 를 계산 하고, 평가점수 $\mathrm{b} 1 \sim \mathrm{b} 15$ 를 활용하여 Level 2 의 평가점수 $\mathrm{c} 1 \sim \mathrm{c} 6$ 을 계산한다. 그리고 평가점수 $\mathrm{c} 1 \sim \mathrm{c} 6$ 을 활용하 여 Level 1에 대한 평가점수를 계산하며, 최종점수는 $\mathrm{d} 1$ $\sim \mathrm{d} 4$ 의 가중산술 평균으로 산출한다.

앞서 살펴본 Table 3 을 통하여 가장 하위 단계인 level 4 평가항목에 대한 종합가중치를 계산 할 수 있다. 그 결 과 Table 5 와 같은 결과가 나타났다. level 4 의 종합가중 치는 level 1 , level 2, level 3, level 4 의 가중치를 곱하여 계산한다. level 4 평가항목의 우선순위는 $\mathrm{HACCP}$ 검증 및 유효성 평가 (0.279281), 운송(원료 입고 및 제품 출 하) (0.117706), 법규 준수 중점 관리 (0.066392), HACCP 문서화 및 기록 관리 (0.054988), 폐기물 관리 (0.048101) 등의 순으로 나타난다.

이러한 분석 결과를 토대로 PL상품 업체 선정에 우선 적으로 영향을 미치는 평가항목들을 고려하면 더 용이한 $\mathrm{PL}$ 업체 선정이 가능할 것이라 판단한다. 
[Table 3] Relative Weights of factors for evaluation

\begin{tabular}{|c|c|c|}
\hline The name of table & Pairwise comparison factors & Weight \\
\hline Three highest Factors & $\begin{array}{c}\text { Management } \\
\text { Environment } \\
\text { Quality }\end{array}$ & $\begin{array}{l}0.086 \\
0.347 \\
0.566\end{array}$ \\
\hline Management & $\begin{array}{c}\text { Fundamental Law Observance } \\
\text { Law Observance } \\
\text { Morality }\end{array}$ & $\begin{array}{l}0.772 \\
0.152 \\
0.076\end{array}$ \\
\hline Environment & $\begin{array}{c}\text { Working Condition } \\
\text { Health Control } \\
\text { Manufacturing facility and Material Management }\end{array}$ & $\begin{array}{l}0.084 \\
0.314 \\
0.602\end{array}$ \\
\hline Quality & $\begin{array}{c}\text { Quality Control } \\
\text { HACCP System } \\
\text { Quality Management System }\end{array}$ & $\begin{array}{l}0.124 \\
0.806 \\
0.070\end{array}$ \\
\hline Working Condition & $\begin{array}{l}\text { Physical Working Condition } \\
\text { Circular Working Condition }\end{array}$ & $\begin{array}{l}0.152 \\
0.848\end{array}$ \\
\hline Health Control & $\begin{array}{l}\text { Facility Health Control } \\
\text { Worker Health Control } \\
\text { Contamination Control }\end{array}$ & $\begin{array}{l}0.085 \\
0.178 \\
0.737\end{array}$ \\
\hline Manufacturing facility and Material Management & $\begin{array}{c}\text { Facility Management } \\
\text { Raw Material Management }\end{array}$ & $\begin{array}{l}0.159 \\
0.841\end{array}$ \\
\hline Quality Control & $\begin{array}{l}\text { Manufacturing Management } \\
\text { Fault Inspection }\end{array}$ & $\begin{array}{l}0.201 \\
0.799\end{array}$ \\
\hline HACCP System & $\begin{array}{l}\text { HACCP System Development } \\
\text { HACCP System Analysis and Supplementation } \\
\text { HACCP System Assessment and Review }\end{array}$ & $\begin{array}{l}0.077 \\
0.129 \\
0.793\end{array}$ \\
\hline Quality Management System & $\begin{array}{c}\text { Quality Regulation } \\
\text { Record Keeping } \\
\text { Follow-up Management }\end{array}$ & $\begin{array}{l}0.328 \\
0.101 \\
0.571\end{array}$ \\
\hline Physical Working Condition & $\begin{array}{c}\text { Surroundings } \\
\text { Working Area } \\
\text { Floor, Wall, Ceiling } \\
\text { Subsidiary Facility }\end{array}$ & $\begin{array}{l}0.369 \\
0.330 \\
0.201 \\
0.100\end{array}$ \\
\hline Circular Working Condition & $\begin{array}{c}\text { Drainage and Piping } \\
\text { Exit and Passage } \\
\text { Window } \\
\text { Illumanation }\end{array}$ & $\begin{array}{l}0.584 \\
0.181 \\
0.168 \\
0.067\end{array}$ \\
\hline Facility Health Control & $\begin{array}{c}\text { Temperature, Humidity } \\
\text { Ventilation } \\
\text { Insect Proof }\end{array}$ & $\begin{array}{l}0.148 \\
0.628 \\
0.224\end{array}$ \\
\hline Worker Health Control & $\begin{array}{c}\text { Worker Health Care } \\
\text { Individual Health Control }\end{array}$ & $\begin{array}{l}0.734 \\
0.266\end{array}$ \\
\hline Contamination Control & $\begin{array}{c}\text { Process Contamination } \\
\text { Waste Management } \\
\text { Disinfection }\end{array}$ & $\begin{array}{l}0.139 \\
0.599 \\
0.262\end{array}$ \\
\hline Facility Management & $\begin{array}{c}\text { Machinery Management } \\
\text { Refrigeration Plant }\end{array}$ & $\begin{array}{l}0.125 \\
0.875\end{array}$ \\
\hline Raw Material Management & $\begin{array}{c}\text { Transportation } \\
\text { Supply Management } \\
\text { Storage }\end{array}$ & $\begin{array}{l}0.670 \\
0.237 \\
0.093\end{array}$ \\
\hline
\end{tabular}


[Table 3] Relative Weights of factors for evaluation (continued)

\begin{tabular}{|c|c|c|}
\hline The name of table & Pairwise comparison factors & Weight \\
\hline Manufacturing Management & $\begin{array}{l}\text { Facility Inspection } \\
\text { Product Designing } \\
\text { Packing } \\
\text { Water Management }\end{array}$ & $\begin{array}{l}0.565 \\
0.123 \\
0.062 \\
0.250\end{array}$ \\
\hline Fault Inspection & $\begin{array}{l}\text { Product Inspection } \\
\text { Recall Program } \\
\text { Foreign Material } \\
\text { Defective Product }\end{array}$ & $\begin{array}{l}0.085 \\
0.144 \\
0.612 \\
0.159\end{array}$ \\
\hline HACCP System Development & $\begin{array}{l}\text { System Construction } \\
\text { Preceding Requisite } \\
\text { Product Characteristic } \\
\text { Process Flow Diagram }\end{array}$ & $\begin{array}{l}0.532 \\
0.303 \\
0.107 \\
0.058\end{array}$ \\
\hline HACCP System Analysis and Supplementation & $\begin{array}{c}\text { Hazard Analysis } \\
\text { Critical Control Point } \\
\text { Threshold Level } \\
\text { Monitoring } \\
\text { HACCP Correction Measure }\end{array}$ & $\begin{array}{l}0.385 \\
0.281 \\
0.194 \\
0.092 \\
0.048\end{array}$ \\
\hline HACCP System Assessment and Review & $\begin{array}{c}\text { Verification } \\
\text { Documentation } \\
\text { System Examination }\end{array}$ & $\begin{array}{l}0.772 \\
0.152 \\
0.076\end{array}$ \\
\hline Quality Regulation & $\begin{array}{l}\text { Quality Policy and Goals } \\
\text { Quality Manual } \\
\text { Responsibility and Authority } \\
\text { Standard of Goods }\end{array}$ & $\begin{array}{l}0.424 \\
0.197 \\
0.140 \\
0.238\end{array}$ \\
\hline Record Keeping & $\begin{array}{c}\text { Document Control } \\
\text { Maintenance of Record }\end{array}$ & $\begin{array}{l}0.201 \\
0.799\end{array}$ \\
\hline Follow-up Management & $\begin{array}{c}\text { Process Control } \\
\text { Correction Measure } \\
\text { Complaint Handling } \\
\text { Instruction and Training }\end{array}$ & $\begin{array}{l}0.577 \\
0.248 \\
0.105 \\
0.069\end{array}$ \\
\hline
\end{tabular}

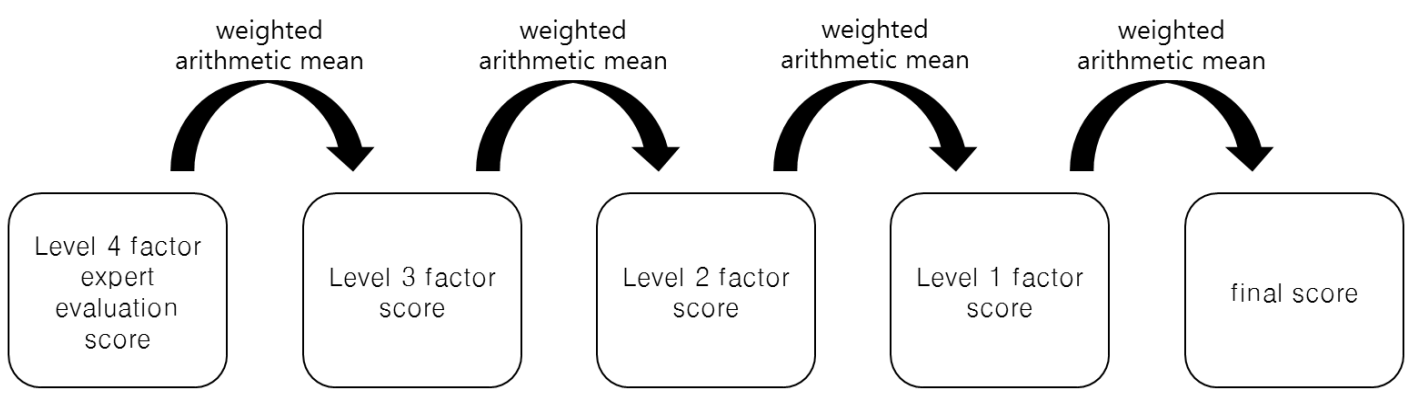

[Fig. 2] The process for score calculation 
[Table 4] An integrated assessment score for selecting a PL product company

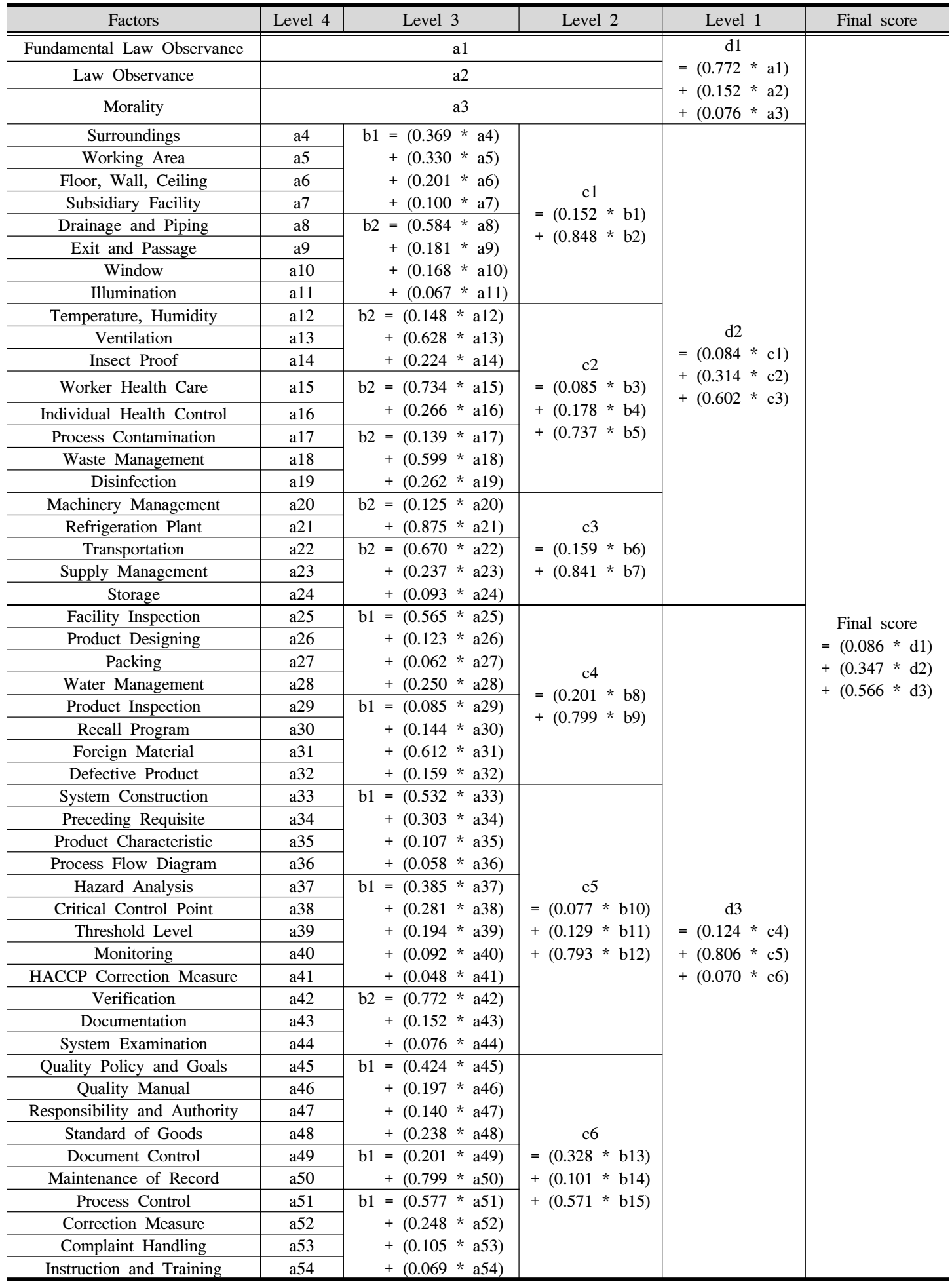


[Table 5] Integrated weights of evaluation elements

\begin{tabular}{|c|c|c|}
\hline Factors & Weight & Ranking \\
\hline Fundamental Law Observance & 0.066392 & 3 \\
\hline Law Observance & 0.013072 & 17 \\
\hline Morality & 0.006536 & 25 \\
\hline Surroundings & 0.001635 & 47 \\
\hline Working Area & 0.001462 & 49 \\
\hline Floor, Wall, Ceiling & 0.000891 & 51 \\
\hline Subsidiary Facility & 0.000443 & 54 \\
\hline Drainage and Piping & 0.014435 & 15 \\
\hline Exit and Passage & 0.004474 & 32 \\
\hline Window & 0.004153 & 33 \\
\hline Illumination & 0.001656 & 46 \\
\hline Temperature, Humidity & 0.001371 & 50 \\
\hline Ventilation & 0.005816 & 26 \\
\hline Insect Proof & 0.002075 & 42 \\
\hline Worker Health Care & 0.014236 & 16 \\
\hline Individual Health Control & 0.005159 & 30 \\
\hline Process Contamination & 0.011162 & 20 \\
\hline Waste Management & 0.048101 & 5 \\
\hline Disinfection & 0.021039 & 11 \\
\hline Machinery Management & 0.004152 & 34 \\
\hline Refrigeration Plant & 0.029062 & 8 \\
\hline Transportation & 0.117706 & 2 \\
\hline Supply Management & 0.041636 & 6 \\
\hline Storage & 0.016338 & 14 \\
\hline Facility Inspection & 0.00797 & 24 \\
\hline Product Designing & 0.001735 & 45 \\
\hline Packing & 0.000875 & 52 \\
\hline Water Management & 0.003527 & 36 \\
\hline Product Inspection & 0.004767 & 31 \\
\hline Recall Program & 0.008075 & 23 \\
\hline Foreign Material & 0.034319 & 7 \\
\hline Defective Product & 0.008916 & 22 \\
\hline System Construction & 0.018688 & 12 \\
\hline Preceding Requisite & 0.010644 & 21 \\
\hline Product Characteristic & 0.003759 & 35 \\
\hline Process Flow Diagram & 0.002037 & 43 \\
\hline Hazard Analysis & 0.022657 & 10 \\
\hline Critical Control Point & 0.016537 & 13 \\
\hline Threshold Level & 0.011417 & 19 \\
\hline Monitoring & 0.005414 & 29 \\
\hline HACCP Correction Measure & 0.002825 & 39 \\
\hline Verification & 0.279281 & 1 \\
\hline Documentation & 0.054988 & 4 \\
\hline System Examination & 0.027494 & 9 \\
\hline Quality Policy and Goals & 0.00551 & 28 \\
\hline Quality Manual & 0.00256 & 40 \\
\hline Responsibility and Authority & 0.001819 & 44 \\
\hline Standard of Goods & 0.003093 & 38 \\
\hline Document Control & 0.000804 & 53 \\
\hline Maintenance of Record & 0.003197 & 37 \\
\hline Process Control & 0.013053 & 18 \\
\hline Correction Measure & 0.005611 & 27 \\
\hline Complaint Handling & 0.002375 & 41 \\
\hline Instruction and Training & 0.001561 & 48 \\
\hline
\end{tabular}

\section{4 일관성 검증}

Table 6는 일관성 검증 결과로 제시된 사례에 대한 $\mathrm{CR}$ 지표 산출 결과 25 개 그룹에서 모두 0.2 이하의 수치가 나타남에 따라 설문조사 결과가 타당함을 확인하였다.

[Table 6] Consistency ratio of the survey

\begin{tabular}{c|c}
\hline The name of table & $\begin{array}{c}\text { Consistency } \\
\text { atio }\end{array}$ \\
\hline \hline Three highest Factors & 0.013 \\
\hline Management & 0.037 \\
\hline Environment & 0.003 \\
\hline Quality & 0.094 \\
\hline Working Condition & 0.000 \\
\hline Health Control & 0.095 \\
\hline Qunufacturing facility and Material Management & 0.000 \\
\hline HACCP System & 0.000 \\
\hline Quality Management System & 0.040 \\
\hline Physical Working Condition & 0.061 \\
\hline Circular Working Condition & 0.132 \\
\hline Facility Health Control & 0.041 \\
\hline Worker Health Control & 0.105 \\
\hline Contamination Control & 0.000 \\
\hline Facility Management & 0.031 \\
\hline Raw Material Management & 0.000 \\
\hline Manufacturing Management & 0.099 \\
\hline Fault Inspection & 0.085 \\
\hline HACCP System Development & 0.011 \\
\hline HACCP System Analysis and Supplementation & 0.038 \\
\hline RACCP System Assessment and Review & 0.041 \\
\hline Record Keeping & 0.037 \\
\hline Hality Regulation & 0.103 \\
\hline Hanagement & 0.000 \\
\hline Hap Manal & 0.084 \\
\hline
\end{tabular}

\section{4. 평가모델의 검증}

본 연구를 통해 제안하는 평가모델과 기존의 단순합산 방식과의 차이점을 검증하기 위해 평가사례 예시에 대한 분석을 수행한다. 기존의 실 산업에서 활용하는 단순합산 방식은 각 평가 항목에 대해 5 단계 등급으로 평가하여 합 산하고, 최종 평가점수를 총 1000점으로 환산한다. 평가 사례 예시에서는 단순합산방식으로 평가할 경우 최종 평 가점수가 유사한 세 후보 업체 $\mathrm{A}, \mathrm{B}, \mathrm{C}$ 를 가정한다. 제안 하는 평가모델과 단순합산방식의 최종 평가점수 산정 결 과는 Table 7과 같다. 두 가지 방법론에 대한 점수 산정 과정은 Table 8, Table 9, Table 10 에 각각 제시되어 있다.

[Table 7] Pilot-study result

\begin{tabular}{c|c|c|c}
\hline & $\begin{array}{c}\text { Company } \\
\text { A }\end{array}$ & $\begin{array}{c}\text { Company } \\
\text { B }\end{array}$ & $\begin{array}{c}\text { Company } \\
\text { C }\end{array}$ \\
\hline \hline Simple Summation & 792.6 & 807.4 & 796.3 \\
\hline Proposed Model & 790.8 & 748.2 & 839.7 \\
\hline
\end{tabular}


[Table 8] Comparison between simple summation method and the proposed model of Company A

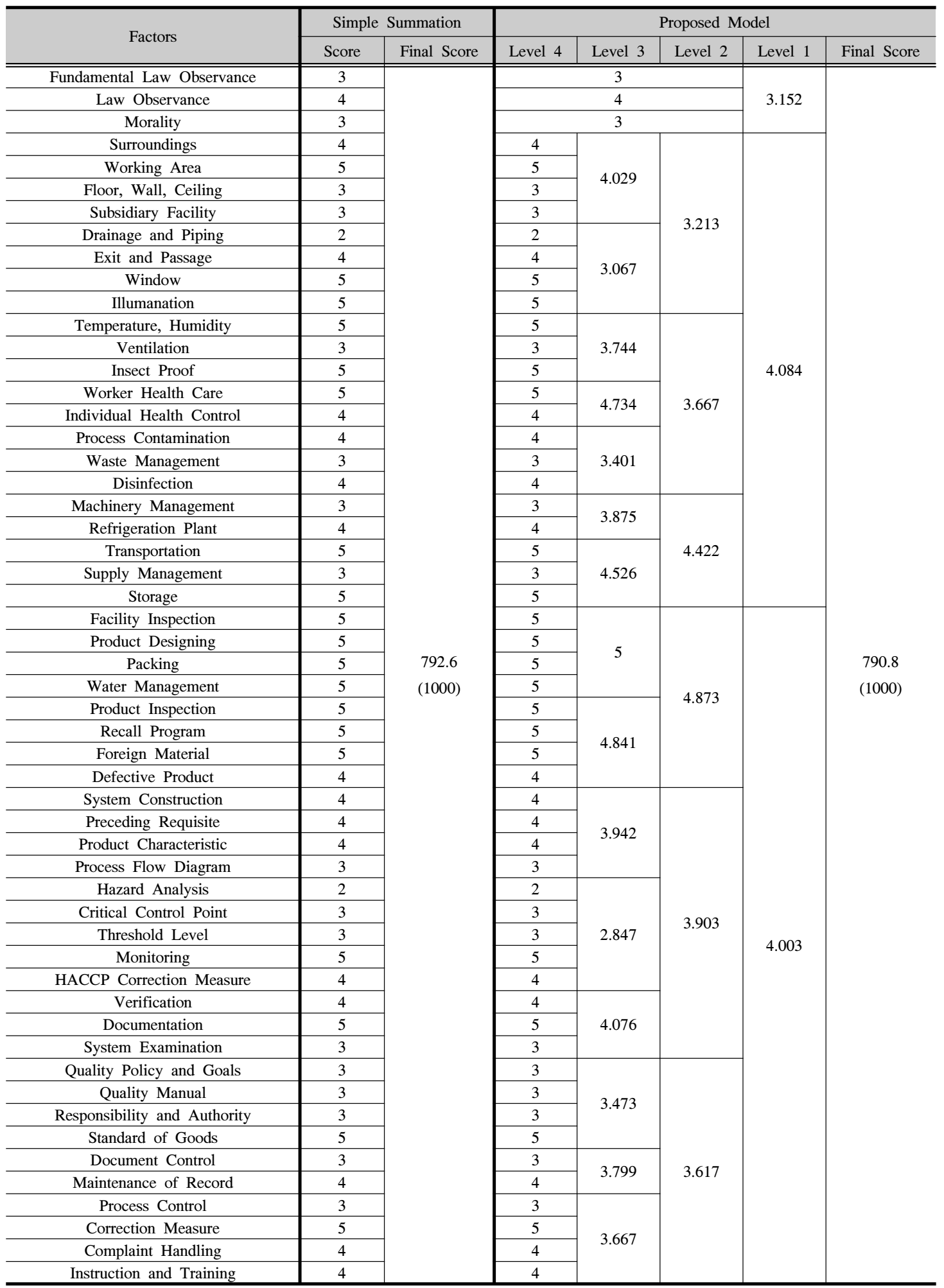


[Table 9] Comparison between simple summation method and the proposed model of Company B

\begin{tabular}{|c|c|c|c|c|c|c|c|}
\hline \multirow{2}{*}{ Factors } & \multicolumn{2}{|c|}{ Simple Summation } & \multicolumn{5}{|c|}{ Proposed Model } \\
\hline & Score & Final Score & Level 4 & Level 3 & Level 2 & Level 1 & Final Score \\
\hline Fundamental Law Observance & 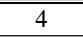 & & \multicolumn{3}{|c|}{4} & \multirow{3}{*}{3.924} & \\
\hline Law Observance & 4 & & & 4 & & & \\
\hline Morality & 3 & & \multicolumn{3}{|c|}{3} & & \\
\hline Surroundings & 4 & & 4 & \multirow{4}{*}{4.33} & \multirow{8}{*}{4.306} & \multirow{21}{*}{3.475} & \\
\hline Working Area & 5 & & 5 & & & & \\
\hline Floor, Wall, Ceiling & 4 & & 4 & & & & \\
\hline Subsidiary Facility & 4 & & 4 & & & & \\
\hline Drainage and Piping & 5 & & 5 & \multirow{4}{*}{4.302} & & & \\
\hline Exit and Passage & 3 & & 3 & & & & \\
\hline Window & 3 & & 3 & & & & \\
\hline Illumanation & 5 & & 5 & & & & \\
\hline Temperature, Humidity & 4 & & 4 & \multirow{3}{*}{3.372} & \multirow{8}{*}{4.313} & & \\
\hline Ventilation & 3 & & 3 & & & & \\
\hline Insect Proof & 4 & & 4 & & & & \\
\hline Worker Health Care & 3 & & 3 & \multirow{2}{*}{3} & & & \\
\hline Individual Health Control & 3 & & 3 & & & & \\
\hline Process Contamination & 5 & & 5 & & & & \\
\hline Waste Management & 5 & & 5 & 4.738 & & & \\
\hline Disinfection & 4 & & 4 & & & & \\
\hline Machinery Management & 3 & & 3 & 3 & & & \\
\hline Refrigeration Plant & 3 & & 3 & 3 & & & \\
\hline Transportation & 3 & & 3 & & 2.922 & & \\
\hline Supply Management & 3 & & 3 & 2.907 & & & \\
\hline Storage & 2 & & 2 & & & & \\
\hline Facility Inspection & 3 & & 3 & \multirow{4}{*}{3.247} & \multirow{8}{*}{3.861} & \multirow{30}{*}{4.692} & \\
\hline Product Designing & 4 & & 4 & & & & \\
\hline Packing & 5 & \multirow{28}{*}{$\begin{array}{c}796.3 \\
(1000)\end{array}$} & 5 & & & & 839.7 \\
\hline Water Management & 3 & & 3 & & & & $(1000)$ \\
\hline Product Inspection & 4 & & 4 & & & & \\
\hline Recall Program & 3 & & 3 & 4.015 & & & \\
\hline Foreign Material & 4 & & 4 & 4.015 & & & \\
\hline Defective Product & 5 & & 5 & & & & \\
\hline System Construction & 5 & & 5 & & & & \\
\hline Preceding Requisite & 4 & & 4 & 4483 & & & \\
\hline Product Characteristic & 3 & & 3 & 4.483 & & & \\
\hline Process Flow Diagram & 5 & & 5 & & & & \\
\hline Hazard Analysis & 5 & & 5 & & & & \\
\hline Critical Control Point & 4 & & 4 & & 4919 & & \\
\hline Threshold Level & 5 & & 5 & 4.718 & & & \\
\hline Monitoring & 5 & & 5 & & & & \\
\hline HACCP Correction Measure & 5 & & 5 & & & & \\
\hline Verification & 5 & & 5 & & & & \\
\hline Documentation & 5 & & 5 & 5 & & & \\
\hline System Examination & 5 & & 5 & & & & \\
\hline Quality Policy and Goals & 4 & & 4 & & & & \\
\hline Quality Manual & 3 & & 3 & 3939 & & & \\
\hline Responsibility and Authority & 5 & & 5 & & & & \\
\hline Standard of Goods & 4 & & 4 & & & & \\
\hline Document Control & 5 & & 5 & 5 & 3548 & & \\
\hline Maintenance of Record & 5 & & 5 & 5 & 3.548 & & \\
\hline Process Control & 3 & & 3 & & & & \\
\hline Correction Measure & 3 & & 3 & 3066 & & & \\
\hline Complaint Handling & 3 & & 3 & & & & \\
\hline Instruction and Training & 4 & & 4 & & & & \\
\hline
\end{tabular}


[Table 10] Comparison between simple summation method and the proposed model of Company C

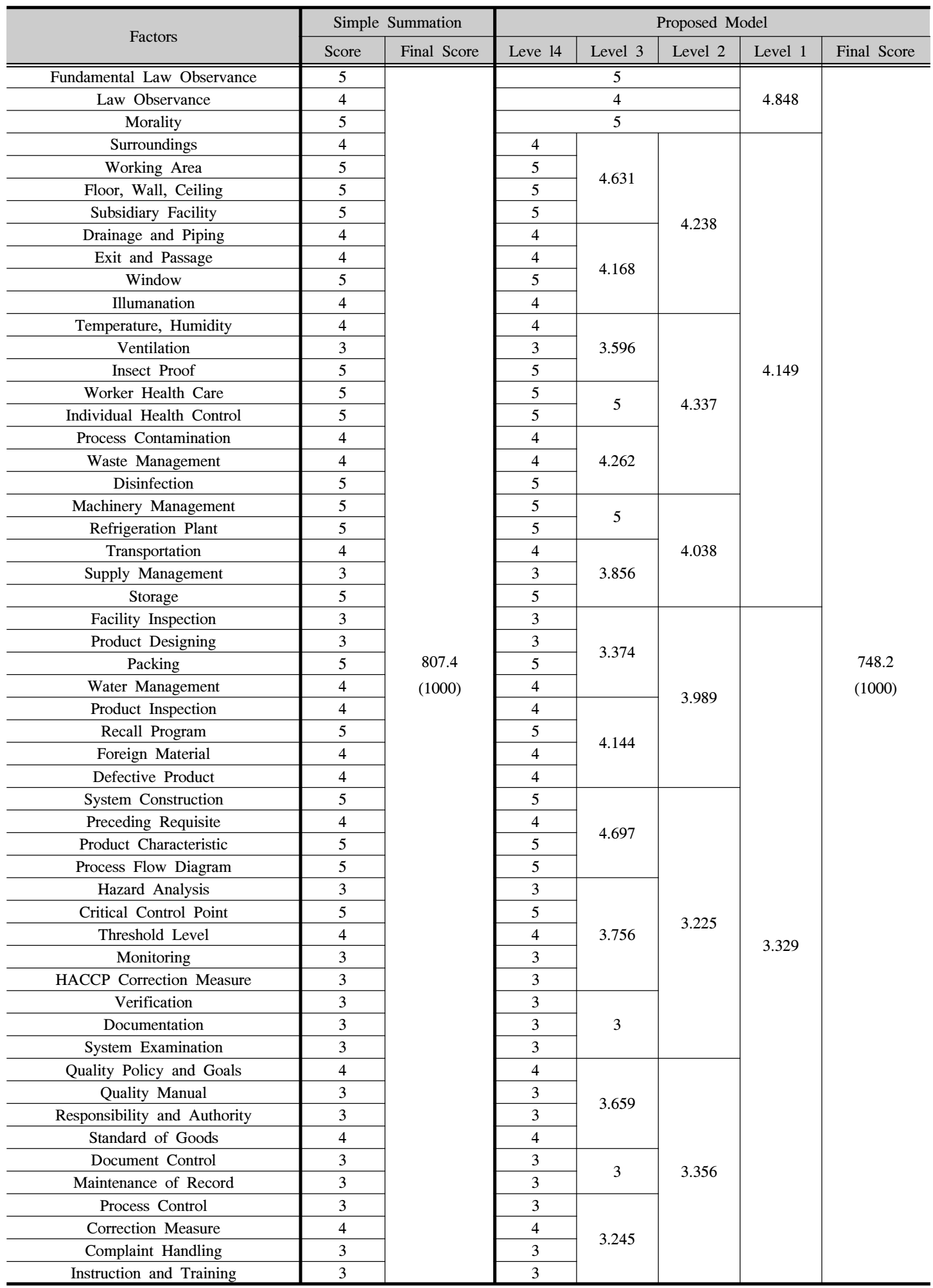


단순합산방식으로 산출한 최종 평가점수는 후보 업체 A 792.6점, 후보 업체 B 807.4점, 후보 업체 C 796.3점으 로 후보업체 $\mathrm{B}$ 가 가장 높게 나타나고, 제안하는 평가모 델로 산출한 최종 평가점수는 후보 업체 A 790.8점, 후보 업체 B 748.2점, 후보 업체 C 839.7점으로 후보 업체 C가 가장 높게 나타난다. 단순합산방식의 경우 선정 업체 $\mathrm{B}$ 와 후보 업체 $\mathrm{A}, \mathrm{C}$ 와의 차이가 각각 14.8점, 11.1점으로 나타나는 반면, 제안하는 모델의 경우 선정 업체 $\mathrm{C}$ 와 후 보 업체 $\mathrm{A}, \mathrm{B}$ 와의 차이가 각각 48.9점, 91.5점으로 나타 난다. 이와 같은 차이는 각 평가항목 점수의 단순 합계로 이루어지는 기존 방식에서 벗어나, 각 항목의 상대적 가 중치를 반영함에 따른다. 자세히 말해, 후보업체 $\mathrm{B}$ 의 경 우 중요도가 낮은 평가항목에 대하여 상대적으로 높은 점수를 가지지만, 후보 업체 $\mathrm{C}$ 의 경우는 중요도가 높은 평가항목에 대하여 점수가 높음을 의미한다. 이와 같은 결과를 통해 기존방식으로 우위를 판단하기 힘든 대안들 에 대하여 제안하는 모델이 PL상품 업체 선정에 용이한 결과를 제시함을 검증하였다.

\section{5. 결론 및 향후연구}

본 연구는 기존 PL상품 업체 선정에 관한 연구가 미비 했던 점을 감안하여, AHP기법을 이용해 PL상품 업체 선 정을 위한 평가항목에 대한 중요도를 반영하는 정량적 평가모델을 제시함에 의의를 가지고, 이를 실 산업에서 발생하는 문제 해결에 활용할 경우 기존의 단순합산방식 과 비교하여 더욱 정교한 의사결정이 가능할 것으로 기 대한다.

$\mathrm{AHP}$ 기법을 적용하는 과정에서는 국내 대형마트 PL상 품 중 매출 비중이 가장 높은 식품품목 업체선정에 대한 실 평가항목을 활용하였고, 이에 대한 계층화, 전문가 설 문을 통해 각 평가항목에 대한 가중치를 산출하였다. $\mathrm{PL}$ 상품 업체 선정에 우선적으로 영향을 미치는 요인들을 살펴본 결과 $\mathrm{HACCP}$ 검증 및 유효성 평가 (0.279281), 운 송(원료 입고 및 제품 출하) (0.117706), 법규 준수 중점 관리 (0.066392)가 높게 나타났다.

평가 모델의 검증을 위해 최종 평가 점수가 유사한 세 후보 업체에 대해 기존 단순합산방식과 제안하는 평가모 델방식을 비교하여 중요도가 높은 평가항목의 점수에 따 라 PL 상품 업체 선정 결과가 달라짐을 보였다. 기존방식 으로 우위를 판단하기 힘든 대안들에 대하여 제안하는 모델이 PL상품 업체 선정에 용이한 결과를 제시함을 검 증하였다.

이번 연구가 이제 막 시작단계에 있는 국내 PL상품업
체선정 관련연구에 있어 초석이 되길 기대하며, 향후 연 구로는 의사결정 기준들에 대한 상호관련성을 고려하여 모델의 현실 반영도를 향상시킬 수 있을 것으로 기대하 고, 이에 대한 대안으로 ANP(Analytic Network Process) 방법론을 고려하고자 한다.

\section{References}

[1] Stren, L. W., A. Al-Ansary, \& J. T. Brown, "Management in Marketing Challels", Englewood Cliffs, New Jersey Prentice Hall, Inc., 1996.

[2] Boone, L. E., \& D. L. Kurtz, "Contemporary Marketing", Fort Worth, T.X.: Dryden, 1995.

[3] McGoldrick, P. J., "Grocery generics-an extension of the private label concept", European Journal of Marketing, Vol.18, pp.5-24, 1984.

DOI: http://dx.doi.org/10.1108/EUM0000000004760

[4] Kumar, N. and J. B. E. M. Steenkamp, "Private Label Strategy: How to Meet the Store Brand Challenge", Havard Business School Press, Boston, Massachusetts, 2007.

[5] Chang-Hyun Jin, "The Relationship between Consumers Awareness, Image, Perceived Quality toward Private Brand and Brand Loyalty", Journal of Korean academy of business administration, Vol.24, No.6, pp.3461-3480, December, 2011.

[6] Yeong-Geun Park, Chang-Wan Kim, "A Study on Factors Affecting PB(Private Brand) Products Preference", Journal of Korean academy of marketing science, Vol.9, pp.189-201, 2002.

[7] Dunne, D. and C. Narasimhan, "The New Appeal of Private Labels," Harvard Business Review, pp.41-52, 1999.

[8] Góez, M. and N.R. Benito, "Manufacturer's Characteristics that Determine the Choice of Producing Store Brands", European Journal of Marketing, Vol.42, No.1/2, pp.154-177, 2008.

DOI: http://dx.doi.org/10.1108/03090560810840952

[9] Vaidyanathan, R. and P. Aggarwal, "Strategic Brand Alliances: Implications of Ingredient Branding for National and Private Lavel Brands", Journal of Product and Brand Management, Vol.9, No.4, pp.214-228, 2000. DOI: http://dx.doi.org/10.1108/10610420010344013

[10] Gyeongdo-Park, Jinyong-Park, and Seungeun-Jeon, "Successful Alliance Between Private and National Brands : The Moderating Effect of Price and Quality 
Sensitivity", Journal of korea distribution association, Vol.12, No.4, pp.109-125, October, 2007.

[11] Verhoef, P. C., E. J. Nijssen, and L. M. Sloot, "Strategic Reactions of National Brand Manufacturers Towards Private Labels: An Empirical Study in The Netherlands," European Journal of Marketing, Vol.36, No.11/12, pp.1309-1326, 2002.

DOI: http://dx.doi.org/10.1108/03090560210445191

[12] Kumar, N., S. Radhakrishnan, and R.C. Rao, "Private Label Vender Selection in a Supply Chain : Quality and Clientele Effects", Journal of Retailing, Vol.86, No.2, pp.148-158, 2010.

DOI: http://dx.doi.org/10.1016/j.jretai.2010.02.007

[13] Saaty, T. L., "The Analytic Hierarchy Process", Mcgraw-Hill, New York, 1980.

[14] Daegi-Kim and Ogyeong-Kwuan "A Study on the Development of Criteria and Priority Orders for Selecting Third Party Logistics Companies", Journal of korean Operations Research and Management Science Society, Vol.20, No.2, pp.151-163 , November, 2003.

[15] Changi-,Nam and Byeongrae-Kim "A Study on E-Commerce Firms' Selecting Criteria for Small Package Express Service Provider by Using the Analytic Hierarchy Process", Journal of Korea Internet Electronic Commerce Association, Vol.3, No.1, 27p 42p, September, 2008.

[16] Sejong-Choi "A Study on the Selection of Aircraft Engine Overhaul Company using AHP", Journal of korean Operations Research and Management Science Society, Vol.1, pp.88-99, 1998.

[17] Seungryeol-Kim, Huisuk-Jeon and Huisuk-Jung "A study on the selection method of the software developer using AHP", Journal of Korean academy of business administration, Vol.12, No.2, pp.15-30, 1995.

[18] wikipedia. Analytic Hierarchy Process. [cited 2013 March 15] http://ko.wikipedia.org/wiki/\%EA\%B3\%84\% EC\%B8\%B5\%EB\%B6\%84\%EC\%84\%9D\%EB\%B2\%95. (accessed Nov., 18, 2013)

\section{최 소 영(So-Young Choi)}

[준회원]

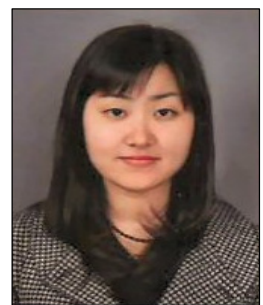

- 2007년 3월 현재 : 고려대학교 산업경영공학과 석사과정

<관심분야>

$\mathrm{SCM}$, 인지모델, 생산 및 물류 정보시스템

\section{김 용 민(Yong-Min Kim)}

[준회원]

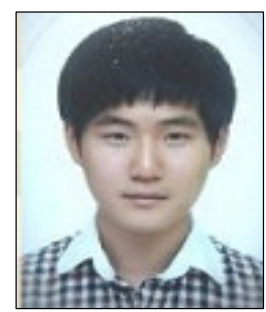

- 2013년 9월 현재 : 고려대학교 산업경영공학과 석사과정

<관심분야>

$\mathrm{SCM}$, 생산 및 물류 정보시스템, 적재 최적화

\section{이 홍 철(Hong-Chul Lee)}

[정회원]

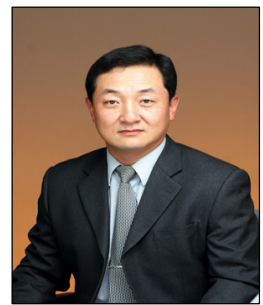

- 1983년 2월 : 고려대학교 산업공 학 학사

- 1988년 2월 : Univ of Texas 산 업공학 석사

- 1993년 2월 : Texas A\&M Univ. 산업공학박사

- 1996년 3월 현재 : 고려대학 교 산업경영공학과 교수

<관심분야>

$\mathrm{SCM}$, 생산 및 물류 정보시스템, PLM 